\title{
The independence of letter identity and letter doubling in reading
}

\author{
Simon Fischer-Baum ${ }^{1}$
}

Published online: 19 August 2016

(C) Psychonomic Society, Inc. 2016

\begin{abstract}
The ability to read requires processing the letter identities in the word and their order, but it is by no means obvious that our long-term memory representations of words spellings consist of only these dimensions of information. The current investigation focuses on whether we process information about another dimension - letter doubling (i.e., that there is a double letter in WEED)-independently of the identity of the letter being doubled. Two experiments that use the illusory word paradigm are reported to test this question. In both experiments, participants are more likely to misperceive a target word with only singleton letters (e.g., WED) as a word with a double (e.g., WEED) when the target is presented with a distractor that contains a different double letter (e.g., WOOD) than when the distractor does not contain a double letter (e.g., WORD). This pattern of results is not predicted by existing computational models of word reading but is consistent with the hypothesis that written language separately represents letter identity and letter doubling information, as previously shown in written language production. These results support a view that the orthographic representations that underlie our ability to read are internally complex and suggest that reading and writing rely on a common level of orthographic representation.
\end{abstract}

Keywords Visual word recognition · Orthography $\cdot$ Models of visual word recognition and priming

Electronic supplementary material The online version of this article (doi:10.3758/s13423-016-1149-8) contains supplementary material, which is available to authorized users.

Simon Fischer-Baum

simon.j.fischer-baum@ rice.edu

1 Department of Psychology, MS-25, Rice University, P.O. Box 1891, Houston, TX 77251, USA
Theories of visual word processing have focused on how we process the letters in a word and their order. The current project investigates whether reading relies on information other than identity and order from written words, specifically, focusing on double letters (e.g., FEED or FOOD). When words with double letters are processed, is letter doubling information processed separately from the identity of that double letter? Figure 1 shows the representation of FEED and FOOD in four theories. In Fig. 1a, word recognition involves identifying individual letters (Davis, 2010; Norris, Kinoshita \& van Casteren, 2010) and double letters are represented by the activation of two adjacent letter identities. In Fig. 1b, word recognition relies on graphemes, or orthographic units that correspond to single phonemes (Perry, Ziegler \& Zorzi, 2013), and each double letter is a specific grapheme. In Fig. 1c, word recognition relies on open bigrams (Grainger et al. 2006) and the double letters are represented by specific units for that letter pair. Critically, none of these theories can capture the fact that FEED and FOOD both include a double letter, even though it is a different letter that is doubled. In contrast in Fig. 1d, word recognition includes a unit (marked Dbl) that, when paired with a letter identity, indicates a double letter. In this way, the representations of FEED and FOOD share a feature, the activation of the Dbl unit, which is not shared with words that do not have a double letter (e.g., FOLD). In the theory depicted in Fig. 1d, there is a separable representation of letter doubling and letter identity. In the theories presented in Fig. 1a-c, there is not.

There is extensive evidence for representations that separate letter identity and doubling in the orthographic representations used in writing (Caramazza \& Miceli, 1990; FischerBaum \& Rapp, 2014; Tainturier \& Caramazza, 1996). For example, Fischer-Baum and Rapp (2014) reported an individual with acquired dyslexia who frequently perseverated letters from one response into the next, for example, spelling "under" 


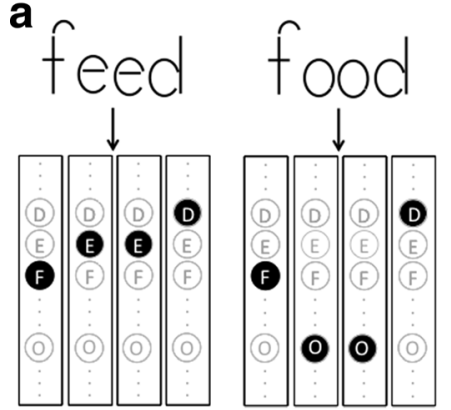

b
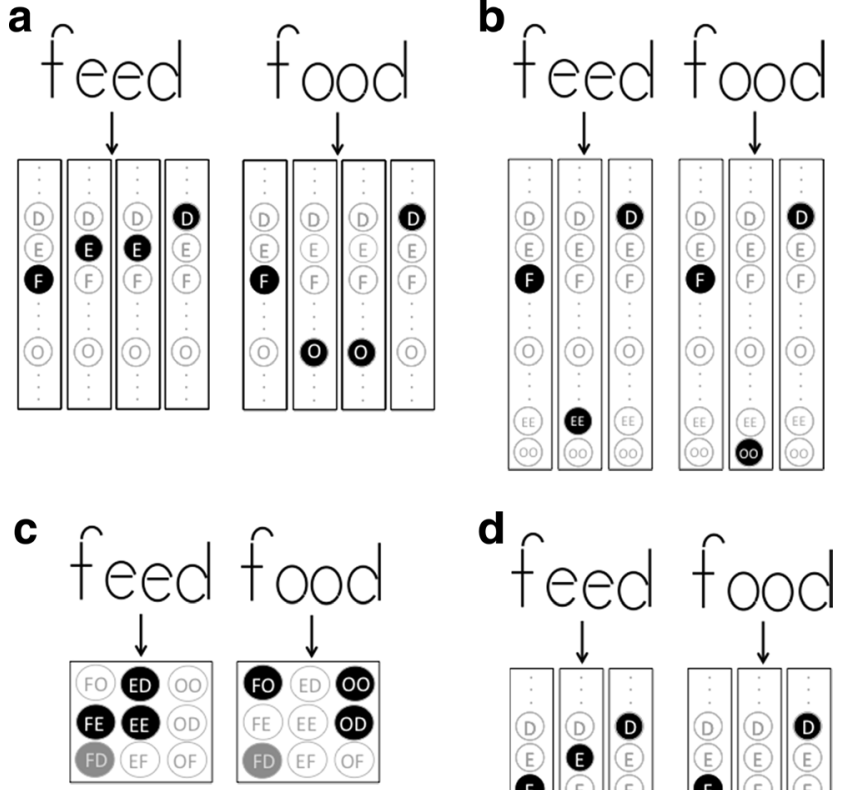

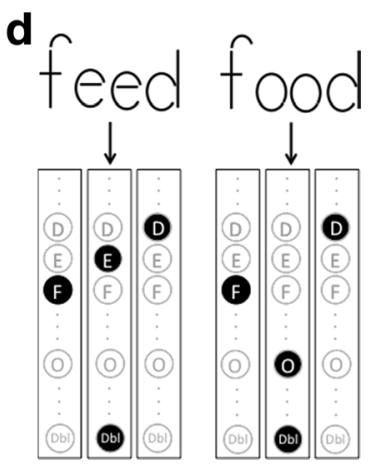

Fig. 1 Orthographic representations of the words FEED and FOOD in four theories of visual word processing. (a) Letter identity and order theories. (b) Grapheme identity and order theories. (c) Open bigram theories. (d) A theory that separates letter identity and letter doubling information

as UNDEL immediately after producing MOLDEL. The patient also occasionally intruded double letters (e.g., spelling "pardon" as PADDY). These doubling errors were preceded by responses that had a different letter doubled (e.g., KIRRY) more often than would be expected by chance. Letter doubling information (i.e., the fact the KIRRY has a double letter) perseverates without the corresponding perseveration of letter identity, suggesting separable doubling and identity information.

The goal of the current project is to determine whether the orthographic representations used in reading similarly separate letter doubling and identity. Following Fischer-Baum and Rapp (2014), this hypothesis is tested by examining whether information that a letter is doubled can move between words independently of the identity of the letter that is doubled. Specifically, the illusory word paradigm is employed to induce these movement errors (Allport, 1977; Davis \& Bowers, 2004, 2006; Fischer-Baum, Charny \& McCloskey, 2011; Treisman \& Souther, 1986). In the illusory word paradigm, participants view brief presentations of word pairs (e.g., STOP and SHIP), which are then masked. Participants are then cued to report one of the words (e.g., SHIP). The cued and noncued words are referred to as the target and distractor, respectively. Participants are more likely to make an error in which one of the letters of the target is substituted (like reporting SHIP as SHOP) when the distractor contains the same letter (e.g., STOP) than when the distractor does not (e.g., STEP). These errors typically are referred to as letter migration errors, with the letter from the distractor moving into the response to the target. Prior research has focused on the conditions under which these letter migration errors occur. The two experiments described use the same paradigm to investigate letter doubling information can migrate between two simultaneously presented stimuli, in the absence of the migration of letter identity information.

\section{Experiment 1}

The first experiment investigated whether participants would make an error in which a target word (e.g., FED) would be reported as a word with the same letter identities, but with one of the letters doubles (e.g., FEED), particularly in the context of a distractor word with a different double letter (e.g., FOOD). If letter doubling information can move independently of letter identity information, these errors are expected. If not, then these errors should occur no more often than expected by chance.

\section{Participants}

Fifty students from Rice University participated in the study for extra course credit. All were native English speakers with normal or corrected-to-normal vision.

\section{Materials}

Table 1 shows examples of the target stimulus, distractor stimuli and doubling error that will be analyzed in this experiment. Thirty word pairs were identified that differed only by the presence of a doubled letter in the interior of the word (e.g., WED - WEED, LITER - LITTER). The word with the singleton (WED, LITER) was designated as the target, and the word with the double letter (WEED, LITTER) was designated as the doubling error. Each target was paired with two distractors that started and ended with the same letters as the target (Davis \& Bowers, 2004) and did not contained the doubled letter that distinguished the target and the doubling error. The double letter distractor contained a different doubled letter in the same position as the double letter in the doubling error (e.g., WOOD for the doubling error WEED, LISSER for LITTER). The control distractor did not contain a doubled letter (e.g., WORD for WEED, LISHER for LITTER). The full set of stimuli is reported in the supplemental materials. For half of the target words, both distractors were real words, and for the other half both were pronounceable pseudowords. Previous illusory word experiments with migrating letters have found minimal effects of the lexical status 
Table 1 Examples of stimuli from Experiments 1 and 2

\begin{tabular}{lllll}
\hline & Condition & Target & Distractor types & Control \\
\cline { 3 - 4 } & & & Double letter & Doubling error \\
\hline Exp. 1 & Word & WED & WOOD & LISHER \\
& Pseudoword & LITER & LISSER & DAPTOR \\
Exp. 2 & Same position & DINER & DAPPOR & DAPUR \\
& Adjacent Position & & DAPOOR & LITTER \\
\hline
\end{tabular}

of distractors on producing these critical errors, implicating a pre-lexical locus of the effect (Treisman \& Souther, 1986). A similar comparison here can be used to identify the locus of the current effect.

To show that existing theories of visual word processing would not predict any difference in doubling errors between the distractor conditions, Colin Davis's Match Calculator tool (www.pc.rhul.ac.uk/staff/c.davis/Utilities/MatchCalc/) was used to assess the similarity between the doubling error and each distractor. Table 2 reports the results of that similarity calculation using two prominent theories of letter position representation - overlapping open bigrams (Grainger et al., 2006) and the SOLAR theory (Davis, 2001). As expected, because these theories do not separate letter identity and doubling, there is no difference in similarity to the doubling error between the distractor conditions; this held for all of the theories assessed by the Match Calculator.

Additional filler trials were included $(n=120)$. In all of the filler trials, the target and the distractor started and ended with the same letter, but none of the stimuli contained a double letter. The fillers ensured that lexical status could not be used to determine which word was the target. Sixty of these trials included a target and a distractor that were both pseudowords, 30 included a target that was a pseudoword and a distractor that was a word, and 30 included a target and a distractor that were both words. In this way, there were an equal number of word and pseudoword targets and distractors across the experiment.

\section{Procedure}

Participants were tested individually. Six practice trials and 180 experimental trials $(30$ critical targets $\times 2$ distractor types and 120 fillers) were presented in a single session that lasted approximately 20 minutes. The experimental trials were divided into two 90-trial blocks, with each target appearing once in each block, and distractor type evenly distributed between the two blocks. Ordering of trials within blocks was random.

Each trial began with a fixation cross displayed at the center of a computer monitor for $1 \mathrm{~s}$. After a 500-ms blank interval, the target and distractor were presented simultaneously for $200 \mathrm{~ms}$ in 24-point lower case Courier font, with one stimulus to the left and the other to the right of the screen's center. Two character spaces separated the last letter of the left stimulus and the first letter of the right stimulus. Stimulus presentation was followed by a single 200-ms non-alphanumeric character mask that covered the screen location of both stimuli. Upon offset of the mask, participants were cued to produce the target stimuli by red crosses that appeared directly overlaying the location in which the target had previously appeared. Responses were recorded and coded by two individuals, blind to the category of the distractor word, for whether the participant produced the correct target, the doubling error, or some other response. Interrater reliability was excellent $(\mathrm{k}=0.90)$, and a third experimenter coded those items on which the two initial coders disagreed.

\section{Results and Discussion}

Trial level coded data for both experiments is available in the Open Science Framework repository (https://osf.io/nvg89/). Table 3 reports the rate of different response types as a function of distractor type. Repeated-measures ANOVAs by participants (F1) and by item (F2) were performed on arcsine transformed percentages of doubling errors, with distractor

Table 2 Similarity between doubling error and distractor by two prevalent theories of orthographic coding, overlapping open bigrams and SOLAR (SD of difference)

\begin{tabular}{|c|c|c|c|c|c|}
\hline & & \multicolumn{2}{|l|}{ OOB } & \multicolumn{2}{|l|}{ SOLAR } \\
\hline & & Double letter & Control & Double letter & Control \\
\hline Exp. 1 & & 0.17 & $10.18(0.07)$ & 0.70 & $0.71(0.03)$ \\
\hline \multirow[t]{2}{*}{ Exp. 2} & Same Pos. & 0.06 & $0.06(0.00)$ & 0.56 & $0.56(0.00)$ \\
\hline & Adj. Pos. & 0.07 & $0.07(0.03)$ & 0.57 & $0.56(0.02)$ \\
\hline
\end{tabular}


Table 3 Breakdown of percentages of response types for double letter and control distractor contexts in experiment 1

\begin{tabular}{llllll}
\hline Response type & \multicolumn{2}{l}{ Word distractors } & & \multicolumn{2}{l}{ Pseudoword distractors } \\
\cline { 2 - 3 } \cline { 6 - 6 } \cline { 6 - 6 } & Double letter & Controls & & Double letter & Controls \\
\hline Correct & 78.9 & 79.3 & & 75.3 & 76.8 \\
Doubling error & 6.0 & 3.5 & & 8.4 & 6.7 \\
Other responses & 15.1 & 17.2 & & 16.3 & 16.5 \\
\hline
\end{tabular}

type (double letter vs. control) and lexical status of distractor (word vs. pseudoword) as factors. By participant, there was a significant effect of lexical status $(\mathrm{F}(1,49)=10.2, p<0.01)$, with a higher overall rate of doubling errors with pseudoword $(7.5 \%)$ than word distractors $(4.7 \%)$. Critically, there was also a significant effect of distractor type $(\mathrm{F}(1,49)=18.9, p<0.001$ ), and no interaction between distractor type and lexical status $(F(1,49)<1)$. Doubling errors were more common with the double letter distractors than with the control distractors, as would be predicted if the doubling feature could migrate independently of letter identity information. However, by item, neither of the main effects nor the interaction was significant $(\mathrm{F}(1,28)<1)$, suggesting that this result is only marginally significant

\section{Experiment 2}

The goal of the second experiment was two-fold. The first was to replicate Experiment 1, given that those results were significant by participant but not by item. The second was to investigate the position specificity of the doubling feature. In the previous experiment, the positions of the double letter in the distractor matched the position in the doubling error (e.g., for both WOOD and WEED the double letters are the second and third letters in the word). In previous work with the illusory word paradigm, it has been shown that distractor letters are more likely to migrate to the matching target position than to a nonmatching position (e.g., SHIP reported as SHOP, given the distractor STOP). However, migrations to approximately matching positions (e.g., SHIP reported as SHOP, given the distractor SOAP) also occur more than chance, providing evidence against position-specific letter coding schemes (Davis \& Bowers, 2004, 2006; Fischer-Baum, Charny \& McCloskey, 2011). The current experiment investigates the same issue with the doubling feature.

\section{Participants}

Thirty-four from Rice University participated in the study for extra course credit. All were native English speakers with normal or corrected-to-normal vision.

\section{Materials}

Twenty-four word pairs were identified that different only by the presence of a doubled letter in the interior of the word (e.g., target DINER with doubling error DINNER). Table 1 shows how target stimulus, doubling errors, and distractor stimuli were constructed from these word pairs in this experiment. Each target was paired with four pseudoword distractors: a "same position" distractor, in which a different double letter appears in the same position of the distractor as it appears in the doubling error (e.g., DAPPOR); an "adjacent position" distractor, in which a different double letter appears in the adjacent position in the distractor (e.g., DAPOOR); and corresponding control distractors without double letters (e.g., DAPTOR and DAPOUR). The full set of stimuli is reported in the supplemental materials. Table 2 reports the similarity between the double letter and control distractors by the overlapping open bigrams (Grainger et al., 2006) and the SOLAR theories (Davis, 2001). As before, there is no difference in similarity to the doubling error between the distractor conditions.

Additional filler trials were included $(\mathrm{n}=96)$. Twenty-four pseudoword targets were presented four times with a different word target each time. In all filler trials, the target and the distractor started and ended with the same letter, but none of these stimuli included a double letter. The fillers were designed so that there were an equal number of word and pseudoword targets across the experiment, and all trials included the presentation of one word and one pseudoword.

\section{Procedure}

Six practice trials and 192 experimental trials (24 critical targets $\times$ 4 distractor types and 96 fillers) were presented in a single session lasting approximately 20 minutes. The experimental trials were divided into four 48-trial blocks, with each target appearing once in each block, and distractor type evenly distributed between the four blocks. Ordering of trials within blocks was random. Presentation and response was identical to Experiment 1.

\section{Results and Discussion}

Table 4 presents the rate of different response types as a function of distractor type. Repeated-measures ANOVAs by participants (F1) and by items (F2) were performed on arcsine transformed percentages of doubling errors, with distractor type (double letter vs. control) and position of double letter (same vs. adjacent position) as factors. By participant, there was a significant effect of distractor type $(\mathrm{F}(1,33)=4.2, p<0.05)$, with no main effect of the position of the double letter or an interaction between distractor type and position of double letter $(\mathrm{F}(1,33)<1)$. By item, the main effect of distractor type was significant $(\mathrm{F}(1,23)$ $=5.1, p<0.05)$, but neither the position of double nor the interaction of the two main effects was significant $(\mathrm{F}(1,23<1)$. 
Table 4 Breakdown of percentages of response types for double letter and control distractors for both same position and adjacent position migrations in experiment 2

\begin{tabular}{lllll}
\hline & Same position & \multicolumn{3}{c}{ Adjacent position } \\
\hline Response type & Double letter & Control & Double letter & Control \\
Correct & 64.7 & 63.4 & 63.5 & 63.0 \\
Doubling error & 5.8 & 4.5 & 5.9 & 4.7 \\
Other response & 29.6 & 32.1 & 30.6 & 32.4 \\
\hline
\end{tabular}

Again, these results can be interpreted as evidence of a migrating double letter feature. Furthermore, the result of this experiment suggests that the double letter feature is not strongly bound to a specific position within the word; there was no difference in the rate of these doubling errors with same or adjacent position double letter distractors.

\section{General Discussion}

The results of two illusory word experiments show that participants are more likely to misperceive a target word (e.g., WED) as having a double letter (e.g., WEED) if that word is paired with a distractor word that has a different double letter (e.g., WOOD) than if that distractor word does not (e.g., WORD), even if that doubled letter is not in the same position of the error and distractor (Experiment 2). The effect is small (7.2\% double letter distractor vs. 5.1\% control distractor in Experiment 1, 6.2\% vs. $4.8 \%$ in Experiment 2) and is only significant by participant in Experiment 1 . The fact that effect was not significant by both participant and item in both experiments raises some issues about its reliability. Successful replication need not rely on statistical significance, given the variability of psychological data (Francis, 2013). Here, a similarly sized effect was observed across these two experiments with different participants and different items. The result is significant by both participant and item in one experiment and by participant in another. Therefore, we should conclude that it is a reliable finding.

As discussed, this result is not predicted by prominent theories of visual word recognition. According to these theories, the double letter and control distractors are equally similar to the doubling error (Table 2), leading to the prediction that this response should be equally likely with the two distractors. However, these theories are underspecified at visual levels of letter processing (Chang, Plaut \& Perfetti, 2016 for a richer conception of visual letter representations) and an alternative explanation for the current finding is that there is greater visual similarity to the doubling error for the double letter distractor than the control distractor. That is, it is possible that WED is more likely to be read as WEED in the context of the distractor WOOD than WORD, because the lower case $[\mathrm{e}]$ is more visually similar to [o] than it is to [r]. This issue was addressed in a post-hoc analysis that compared the visual similarity of the letters in each distractor with the letters in the doubling error, based on similarity ratings from Boles and Clifford (1989). No significant differences were observed between the distractor conditions. Furthermore, visual letter similarity did not correlate with the likelihood of producing the doubling error. Therefore, it is unlikely that the effect is due to differences at a level of visual letter processing.

Instead, the current finding can be explained by a theory that involves representations that separate letter identity and doubling, an example of which is shown in Fig. 1d. This separation of identity and doubling could be occurring at a number of levels of representation. The lack of a distractor-word lexicality effect in Experiment 1 implicates prelexical representations but visual word recognition has been argued to rely on several levels of prelexical letter representation. Schubert and McCloskey (2013) propose a distinction between an allographic level that represents distinct visual shapes of letter and an abstract identity letter level that abstracts away from visual properties like case and font. Based on the current work, either level could be the locus of the effect.

Previously work in writing has argued for a distinction between doubling and identity representations at a level of abstract letter identity representation (Caramazza \& Miceli, 1990; Fischer-Baum \& Rapp, 2014). One possibility is that reading and writing share this level of representation, which includes a doubling feature representation. Such an account would explain why similar patterns of doubling errors are observed in reading and writing. Further work is required to computationally specify how reading and writing could rely on this common level of representation, particularly because writing is slow and attention demanding, whereas reading is faster and far more automatic.

In writing, letter doubling information is thought to be specifically represented by a doubling feature, associated with a letter identity when it is immediately repeated (e.g., WEED) but not to letter identities that are repeated nonadjacently in a word (e.g., BERET; Tainturier \& Caramazza, 1996). The current research does not explicitly address the issue of nonadjacent repeated letters in reading, so the representation of these repeated letter words remains an open question. However, if reading and writing rely on common representations, the prediction would be that in reading there also is only a specially representation for immediately repeated letters.

Assuming that orthographic representations in reading separate letter doubling and identity information can help explain several striking observations about written language processing. Double letters play a critical role in morphological decomposition. Many morphologically complex forms in English involve doubling a final consonant in the stem (e.g., hitting, bagged, metallic). Morpho-orthographic decomposition of written words, such as decomposing hitting into the combination of hit and +ing, requires identifying these double letters and reducing them to singletons (McCormick, Rastle \& Davis, 2008). This pattern can be more succinctly explained by assuming cognitive 
operations that reference doubling, not identity information. Furthermore, the fact that doubling information is not bound strongly to word position, as found in Experiment 2, can explain why readers have difficulty recognizing misspellings in which the wrong letter is doubled in the word (e.g., tommorow, parralel; Holmes \& Carruthers, 1998). According to the account above, these misspellings share letter identities and the presence of a double letter feature with the correct spelling, but the double letter feature is associated with a different letter identity in a different position in the word.

Do these results suggest that computational models of visual word processing need to be modified? It depends on the goal of the model. Small additional details, such as incorporating the doubling feature, will likely not contribute to our understanding of phenomena like developmental dyslexia. However, some models are concerned with specific details of representation at each level of the reading system with the goal of predicting experimental results to millisecond precision (Davis, 2010). The current result suggests that these models need to incorporate representations like the doubling feature. Beyond the results of these two experiments, it is likely that orthographic representations are significantly more richly structured that those incorporated into current models, for example containing representations of syllabic structure (Taft \& Krebs-Lazendic, 2013) and consonant-vowel status (Chetail, Drabs \& Content, 2014).

Some might argue that adding these types of structures to models of visual word recognition make them unnecessarily complex. However, it has been independently argued that processing doubling and identity information separately may be a perceptual primitive that underlies many aspects of cognitive processing (Endress, Nespor \& Mehler, 2009). Doubling may play a critical role in acquiring grammatical knowledge (Marcus et al., 1999) and is a common morphological process cross-linguistically (McCarthy \& Prince, 1995). The current results suggest that orthographic representations in reading separate letter doubling from letter identity information. This type of processing may not be specific to reading, instead reflecting a general cognitive constraint.

Acknowledgments Thanks to Lilia Ramos for her work on Experiment 1, Elizabeth Garcia for her work on Experiment 2, and David Kajander for help in coding and a discussion of an earlier draft of the manuscript.

\section{References}

Allport, D. A. (1977). On knowing the meaning of words we are unable to report: The effects of visual masking. In S. Dornic (Ed.), Attention and performance VI (pp. 505-533). Hillsdale, NJ: Erlbaum.

Boles, D. B., \& Clifford, J. E. (1989). An upper-and lowercase alphabetic similarity matrix, with derived generation similarity values. Behavior Research Methods, Instruments, \& Computers, 21(6), 579-586.

Caramazza, A., \& Miceli, G. (1990). The structure of graphemic representations. Cognition, 37(3), 243-297.
Chang, L. Y., Plaut, D. C., \& Perfetti, C. A. (2016). Visual complexity in orthographic learning: Modeling learning across writing system variations. Scientific Studies of Reading, 20(1), 64-85.

Chetail, F., Drabs, V., \& Content, A. (2014). The role of consonant/vowel organization in perceptual discrimination. Journal of Experimental Psychology: Learning, Memory, and Cognition, 40(4), 938-961.

Davis, C. J. (2001). The self-organising lexical acquisition and recognition (SOLAR) model of visual word recognition (Doctoral dissertation, ProQuest Information \& Learning).

Davis, C. J. (2010). The spatial coding model of visual word identification. Psychological Review, 117(3), 713-758.

Davis, C. J., \& Bowers, J. S. (2004). What do letter migration errors reveal about letter position coding in visual word recognition? Journal of Experimental Psychology: Human Perception and Performance, 30(5), 923-941.

Davis, C. J., \& Bowers, J. S. (2006). Contrasting five different theories of letter position coding: Evidence from orthographic similarity effects. Journal of Experimental Psychology: Human Perception and Performance, 32(3), 535-557.

Endress, A. D., Nespor, M., \& Mehler, J. (2009). Perceptual and memory constraints on language acquisition. Trends in Cognitive Sciences, 13(8), 348-353.

Fischer-Baum, S., Charny, J., \& McCloskey, M. (2011). Both-edges representation of letter position in reading. Psychonomic Bulletin \& Review, 18(6)), 1083-1089.

Fischer-Baum, S., \& Rapp, B. (2014). The analysis of perseverations in acquired dysgraphia reveals the internal structure of orthographic representations. Cognitive Neuropsychology, 31(3), 237-265.

Francis, G. (2013). Replication, statistical consistency, and publication bias. Journal of Mathematical Psychology, 57(5), 153-169.

Grainger, J., Granier, J. P., Farioli, F., Van Assche, E., \& van Heuven, W. J. (2006). Letter position information and printed word perception: The relative-position priming constraint. Journal of Experimental Psychology: Human Perception and Performance, 32(4), 865-884.

Holmes, V. M., \& Carruthers, J. (1998). The relation between reading and spelling in skilled adult readers. Journal of Memory and Language, 39(2), 264-289.

Marcus, G. F., Vijayan, S., Rao, S. B., \& Vishton, P. M. (1999). Rule learning by seven-month-old infants. Science, 283(5398), 77-80.

McCarthy, J., \& Prince, A. (1995). Faithfulness and reduplicative identity. In University of Massachusetts occasional papers 18: Papers in Optimality Theory, 249-384. GLSA, University of Massachusetts, Amherst.

McCormick, S. F., Rastle, K., \& Davis, M. H. (2008). Is there a 'fete'in 'fetish'? Effects of orthographic opacity on morpho-orthographic segmentation in visual word recognition. Journal of Memory and Language, 58(2), 307-326.

Norris, D., Kinoshita, S., \& van Casteren, M. (2010). A stimulus sampling theory of letter identity and order. Journal of Memory and Language, 62(3), 254-271.

Perry, C., Ziegler, J. C., \& Zorzi, M. (2013). A computational and empirical investigation of graphemes in reading. Cognitive Science, 37(5), $800-828$.

Schubert, T., \& McCloskey, M. (2013). Prelexical representations and processes in reading: Evidence from acquired dyslexia. Cognitive Neuropsychology, 30(6), 360-395.

Taft, M., \& Krebs-Lazendic, L. (2013). The role of orthographic syllable structure in assigning letters to their position in visual word recognition. Journal of Memory and Language, 68(2), 85-97.

Tainturier, M. J., \& Caramazza, A. (1996). The status of double letters in graphemic representations. Journal of Memory and Language, 35(1), 53-73.

Treisman, A., \& Souther, J. (1986). Illusory words: The roles of attention and of top-down constraints in conjoining letters to form words. Journal of Experimental Psychology: Human Perception and Performance, 12(1), 3-17. 